\title{
Dynamic Character of Human Growth Hormone and Its Receptor: Normal Mode Analysis
}

\author{
Youji Kurihara, ${ }^{a}$ Tomoko Watanabe, ${ }^{a}$ Hiroyuki Nojima, ${ }^{a}$ Mayuko TakedA-Shitaka, ${ }^{a}$ \\ Hiromi Sumikawa, ${ }^{b}$ Kenshu Kamiya, ${ }^{c}$ and Hideaki UmeYamA ${ }^{*, a}$ \\ ${ }^{a}$ School of Pharmaceutical Sciences, Kitasato University; 5-9-1 Shirokane, Minato-ku, Tokyo 108-8641, Japan: ${ }^{b}$ Central \\ Research Laboratories, Ajinomoto Co., Inc.; 1-1 Suzuki-cho, Kawasaki-ku, Kawasaki 210-8681, Japan: and ${ }^{c}$ School of \\ Science, Kitasato University; 1-15-1 Kitasato, Sagamihara, Kanagawa 228-8555, Japan. \\ Received October 30, 2002; accepted April 4, 2003
}

\begin{abstract}
Human growth hormone (hGH) induces dimerization of its binding protein (hGHbp). hGH binds to the first hGHbp (bp1) on site 1, and then the hGH-bp1 heterodimer complex binds to the second hGHbp (bp2) on site 2. Although the interactions of hGH and hGHbps have been studied from different viewpoints, few studies from a dynamic viewpoint have been reported. Especially, since in the SCOP domain database hGHbp is classified as two clear immunoglobulin-like domains, it is of interest to understand how hGH interacts with the hGHbp domains. Therefore, we carried out normal mode analysis (NMA) of free hGH, free bp1, free bp2, and the hGH-bp1 heterodimer complex, as well as the hGH-bp1-bp2 ternary complex to investigate how the dynamics of the proteins change before and after forming the complexes. NMA showed that the domain motion between the $\mathrm{N}$-terminal and the $\mathrm{C}$-terminal domains of free bp1 markedly decreased after binding to $\mathrm{hGH}$, and that the domain motion of bp2 decreased similarly after binding to the hGH-bp1 heterodimer complex. The present study demonstrates that hGH regulates the inter-domain motions of both hGHbps.
\end{abstract}

Key words human growth hormone; human growth hormone binding protein; domain motion; normal mode analysis

Human growth hormone (hGH) is a kind of anterior pituitary hormone, and is necessary for regulation of normal growth and metabolism. ${ }^{1)} \mathrm{hGH}$ has two sites for binding to two molecules of its binding protein (hGHbp) and induces dimerization of hGHbp. ${ }^{2)}$ The dimerization of hGHbp initiates phosphorylation of the Janus tyrosine kinase, Jak2, leading to cell proliferation and other effects. ${ }^{3,4)}$ When the hGH concentration is high for hGHbp, one hGH molecule binds to only one, not two, hGHbp molecules; therefore, hGHbp cannot form its biologically active dimer, and thus the signal transduction does not occur. ${ }^{5,6)}$

X-Ray crystallographic study ${ }^{7}$ shows that hGH binds to the first hGHbp (bp1) at site 1 and to the second hGHbp (bp2) at site 2 (Fig. 1); and, further, that hGH has a four-helix bundle and that three additional short helices are found in the connection loops of the four helices. hGHbp has the N-terminal (residues 32-130) and the C-terminal (residues 131238) like immunoglobulin domains. The division of these two domains is based on the Structural Classification of Proteins (SCOP) database. ${ }^{8,9}$ hGHbp is classified into the cytokine receptor superfamily, including the receptor molecules of erythropoietin (EPO), prolactin (PRL), interleukin (IL)-2, $3,4,5,6$ and 7, granulocyte colony-stimulation factor (G$\mathrm{CSF}$ ) and granulocyte-macrophage colony-stimulation factor (GM-CSF). ${ }^{10,11)}$

Although interactions of hGH and hGHbps have been studied from different viewpoints, few studies from a dynamic viewpoint have been reported. Especially, it is of great interest to know how the two domains classified in the SCOP domain database are dynamically regulated in the interaction with hGH. Hence, we carried out normal mode analysis (NMA). In NMA, protein conformational dynamics are described in terms of a superposition of independent harmonic oscillators called normal modes. NMA is a useful tool for understanding dynamics of protein conformation and analyz- ing small fluctuations around a stable state under the harmonicity of the energy surface. Fluctuations in atoms of a protein will influence its stability and functions. It has been shown that NMA is useful for the study of protein dynamics such as enzyme-inhibitor binding motion, ${ }^{12,13)}$ the gating mechanism of the potassium channel KcsA, ${ }^{14)}$ the antigen peptide binding mechanism to the Class I MHC molecules ${ }^{15}$ and analysis of domain motion in proteins. ${ }^{16-18)}$ According to other published papers, ${ }^{2)}$ bp1 binds to hGH and sequentially, the hGH-bp1 heterodimer complex binds to bp2. Therefore, we investigated the dynamic behavior of hGH, bp 1 and bp 2 in these free states, and also in as well as in the actual complex states.

\section{Experimental}

Protein Initial Coordinate The X-ray structure coordinates of the complex hGH and hGHbps were obtained from the Protein Data Bank (PDB), ${ }^{19}$ (PDB code 3HHR). ${ }^{7)}$ In 3HHR, chain A (residues 1-190) is hGH, chain B (residues 32-234) is bp1 and chain C (residues 32-236) is bp2. Some residues, which were disordered and not included in 3HHR, were modeled using BIOCES[E]..$^{20,21)}$ The residues $1-31$ of hGHbps were not modeled because their coordinates were not given in 3HHR.

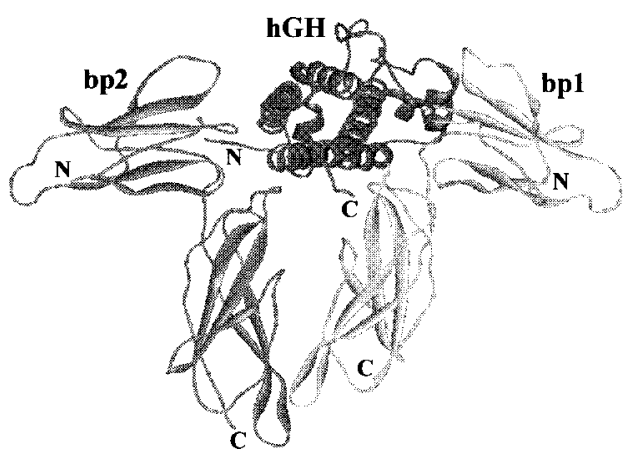

Fig. 1. X-Ray Crystal Structure of the hGH-bp1-bp2 Ternary Complex ${ }^{7)}$ The $\mathrm{N}$ - and C-terminals are marked $\mathrm{N}$ and $\mathrm{C}$, respectively. 
Normal Mode Analysis NMA was carried out using the method described in earlier reports from our laboratory ${ }^{13,15,22)}$ Before NMA, the molecular structures were energetically optimized with a slightly modified force field of $\mathrm{AMBER}^{23)}$ and a threshold of $0.04 \mathrm{kcal} / \mathrm{mol} \AA$ for the maximal component of atomic gradients. ${ }^{15)}$ In our calculations, we assumed that the protein molecules were in vacuo, but that a distance-dependent dielectric constant $(r / \AA)$ for electrostatic energy was maintained. ${ }^{15)}$ The electrostatic potential and the van der Waals potential were cut off at $9.0 \AA$, and were switched smoothly and continuously to a value of zero at $10.0 \AA$. Energy minimization was performed with Cartesian coordinates, and the NMA was done with torsion angles except for the peptide $\mathrm{C}-\mathrm{N}$ bond. The fluctuations of the atoms were calculated by assuming a temperature of $300 \mathrm{~K}$ using normal modes and vibrational frequencies.

To study the dynamics changes of hGH, bp1 and bp2 before and after forming the complexes, NMA was carried out with hGH and bp1 under three states, free, the hGH-bp1 heterodimer complex and the hGH-bp1-bp2 ternary complex, and with bp2 under two states, free and the hGH-bp1-bp2 ternary complex. The coordinates of free $\mathrm{hGH}$, free bp1, free bp2 and the hGH-bp1 heterodimer complex were obtained by removing other molecules from the coordinates of the hGH-bp1-bp2 ternary complex (3HHR), because the X-ray structures of free hGHbps and the hGH-bp1 heterodimer complex of the wild type have yet to be resolved, and mutation studies of the hGH-bp1 heterodimer complex demonstrated that there was little structural change in forming the hGH-bp1-bp2 ternary complex..$^{24,25)}$ For calculations of the complexes, relative motions (translation and rotation) between constituent molecules were also included. In comparisons of the protein molecules before and after forming the complexes, the target proteins in the complexes were applied to Eckart's condition ${ }^{26)}$ to separate external and internal motions. ${ }^{12,15)}$ Fluctuations of ten energetically optimized structures under different energy-converging conditions were determined by averaging, since the results of the atomic fluctuations were slightly different depending on the
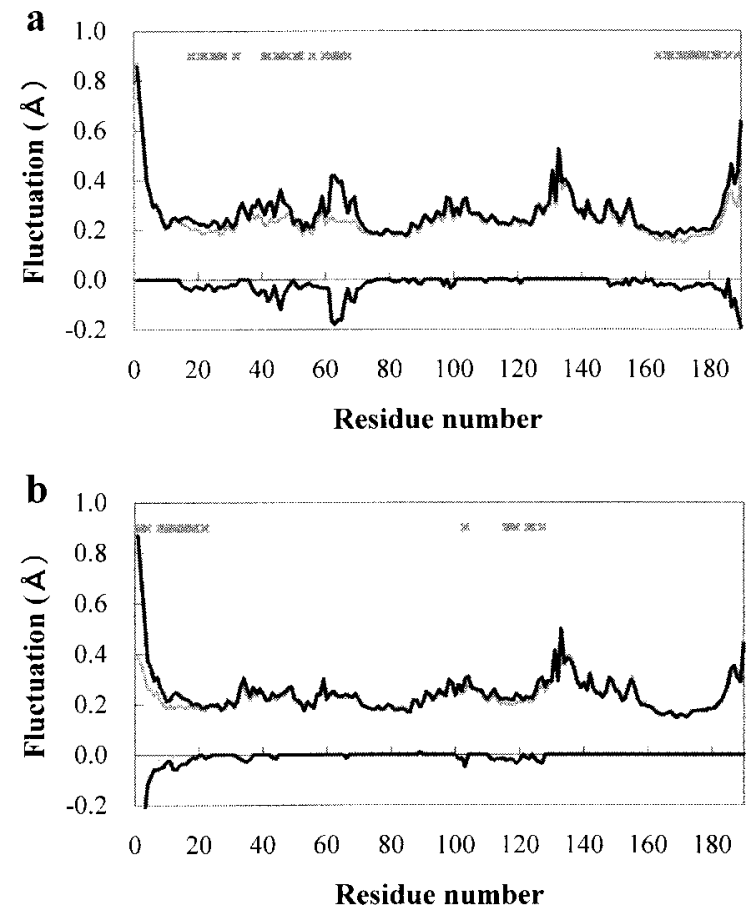

Fig. 2. Fluctuations of $\mathrm{C} \alpha$ Atoms of hGH in Free Form, in the hGH-bp1 Heterodimer Complex and in the hGH-bp1-bp2 Ternary Complex

(a) Thick and thin lines above zero show fluctuations of $\mathrm{C} \alpha$ atoms of hGH in free form and in the hGH-bp1 heterodimer complex, respectively. Bottom line shows significant differences of fluctuations between free form (thick line) and the hGH-bp1 heterodimer complex (thin line). Top cross marks show contact residues of hGH with bp1 in the hGH-bpl heterodimer complex. (b) Thick and thin lines above zero show fluctuations $\mathrm{C} \alpha$ atoms of hGH in the hGH-bpl heterodimer complex and in the hGH-bp1-bp2 ternary complex, respectively. Bottom line shows significant differences of fluctuations between the hGH-bp1 heterodimer complex (thick line) and the hGH-bp1-bp2 ternary complex (thin line). Top cross marks show contact residues of hGH with bp2 in the hGH-bp1-bp2 ternary complex. In (a) and (b), the fluctuations are averages of ten locally minimum structures prepared by energy minimization under different energy-converging conditions, the significant differences are calculated using the Wilcoxon rank sum test, and the contact residues are calculated using MSAS. ${ }^{29)}$ local minimum structure. The differences of fluctuations between the free and the complex forms were examined by the Wilcoxon sum test, a non parametric test.

\section{Results and Discussion}

Fluctuations of hGH Fluctuations of $\mathrm{C} \alpha$ atoms of hGH in free form, the hGH-bp1 heterodimer complex and the hGH-bp1-bp2 ternary complex are shown in Fig. 2. After binding to free bp1 of free hGH, the fluctuation of hGH significantly decreased at residues $37-49,51-75$ and the Cterminal region in contact regions with bp1 (Fig. 2a). After binding to free bp2 of the hGH-bp1 heterodimer complex,

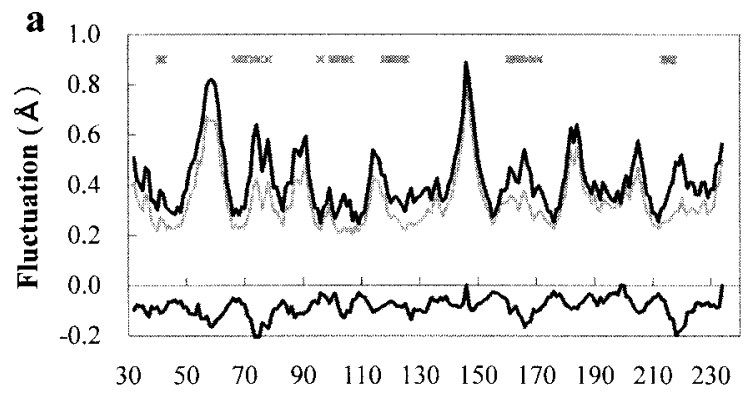

Residue number
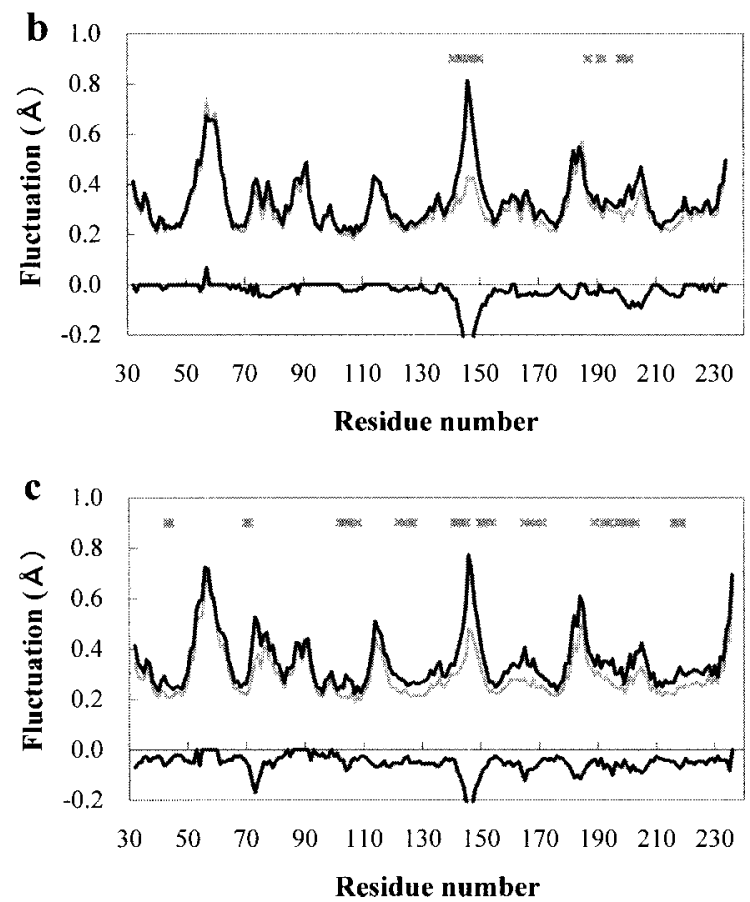

Fig. 3. Fluctuations of $\mathrm{C} \alpha$ Atoms of hGHbps in Free hGHbps, in the hGH-bp1 Heterodimer Complex and in the hGH-bp1-bp2 Ternary Complex in a Case Where the Center of Gravity is on the Whole Domain

(a) Thick and thin lines above zero show fluctuations of $\mathrm{C} \alpha$ atoms of bp1 in free form and in the hGH-bp1 heterodimer complex, respectively. Bottom line shows significant differences of fluctuations between free form (thick line) and the hGH-bp1 heterodimer complex (thin line). Top cross marks show contact residues of bp1 with hGH in the hGH-bp1 heterodimer complex. (b) Thick and thin lines above zero show fluctuations $\mathrm{C} \alpha$ atoms of bp1 in the hGH-bp1 heterodimer complex and the hGH-bp1-bp2 ternary complex, respectively. Bottom line shows significant differences of fluctuations between the hGH-bp1 heterodimer complex (thick line) and in the hGH-bp1-bp2 ternary complex (thin line). Top cross marks show contact residues of bp1 with bp2 in the hGH-bp1-bp2 ternary complex. (c) Thick and thin lines above zero show fluctuations of $\mathrm{C} \alpha$ atoms of bp2 in free form and in the hGH-bp1-bp2 ternary complex, respectively. Bottom line shows significant differences of fluctuations between free form (thick line) and the hGH-bp1-bp2 ternary complex (thin line). Top cross marks show contact residues of bp2 with hGH and bp1 in the hGH-bp1-bp2 ternary complex. In (a), (b) and (c), the fluctuations, the significant differences and the contact residues are calculated in the same way as those of Fig. 2. 
the fluctuation of hGH also decreased at the N-terminal region in the contact region with bp2 (Fig. 2b). These results showed that the fluctuations of hGH decreased only in the contact regions with hGHbps.

Fluctuations of hGHbps Fluctuations of $\mathrm{C} \alpha$ atoms of $\mathrm{bp} 1$ in free form, the hGH-bp1 heterodimer complex and the hGH-bp1-bp2 ternary complex, and of bp2 in free form and the hGH-bp1-bp2 ternary complex are shown in Fig. 3. In consideration of the selectivity by which free hGH initially binds to free $\mathrm{bp} 1$ to form the hGH-bp1 heterodimer complex and the hGH-bp1 heterodimer complex additionally binds to free $b p 2$ to form the hGH-bp1-bp2 ternary complex, the fluctuations of hGHbps before and after forming the complexes were compared. After binding to free hGH of free bp1, the fluctuation of $b p 1$ decreased at almost all residues (Fig. 3a), but after binding to free bp 2 of the hGH-bp1 heterodimer complex, the fluctuation of $b p 1$ mainly decreased at residues $143-152$ and at about 200 in the contact regions with bp2 (Fig. 3b). The differences of the fluctuations were positive at residues $55-60$. We modeled residues around
55-60 that were excluded in X-ray structure coordinates, because these were disordered in the crystal structure. As a result, compared with fluctuations around other residues, the fluctuations at residues 55-60 were unusual. Upon free bp2 bound to the hGH-bp1 heterodimer, the fluctuation of $b p 2$ decreased at almost all residues (Fig. 3c). The value of the average fluctuations of free $b p 1$ was different from those of free bp2 (Figs. 3a, c). The structures of free bp1 and free bp2 were obtained by removing hGH-bp2 and hGH-bp1 from Xray structure coordinates, respectively. The root mean square deviation ( $\mathrm{rms}$ ) for superposition between the main chain atoms in free bp 1 and in free bp 2 was $1.45 \AA$ and the average rms among the main chain atoms in 10 optimized free bp 1 and 10 optimized free bp 2 structures was $2.12 \AA$. The fluctuations of free $b p 1$ and free $b p 2$ were different from each other probably due to the different structures. Although the average fluctuations of free bp1 were different from those of free $b p 2$, the shapes of fluctuations of free $b p 1$ were very similar to those of free bp2 and the fluctuations of free bp1 and free bp2 are larger than these of hGH-bp1 and hGH-
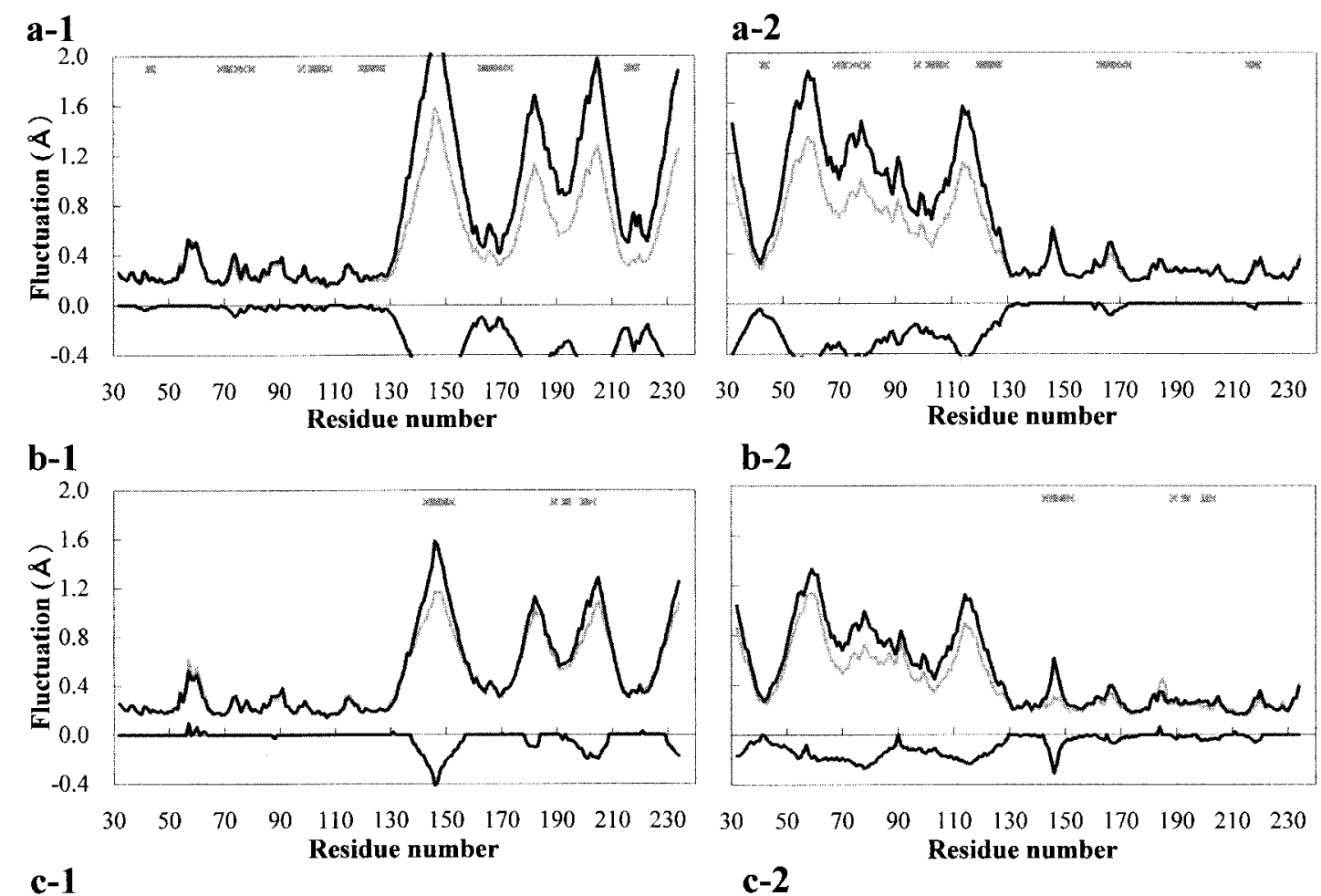

b-2
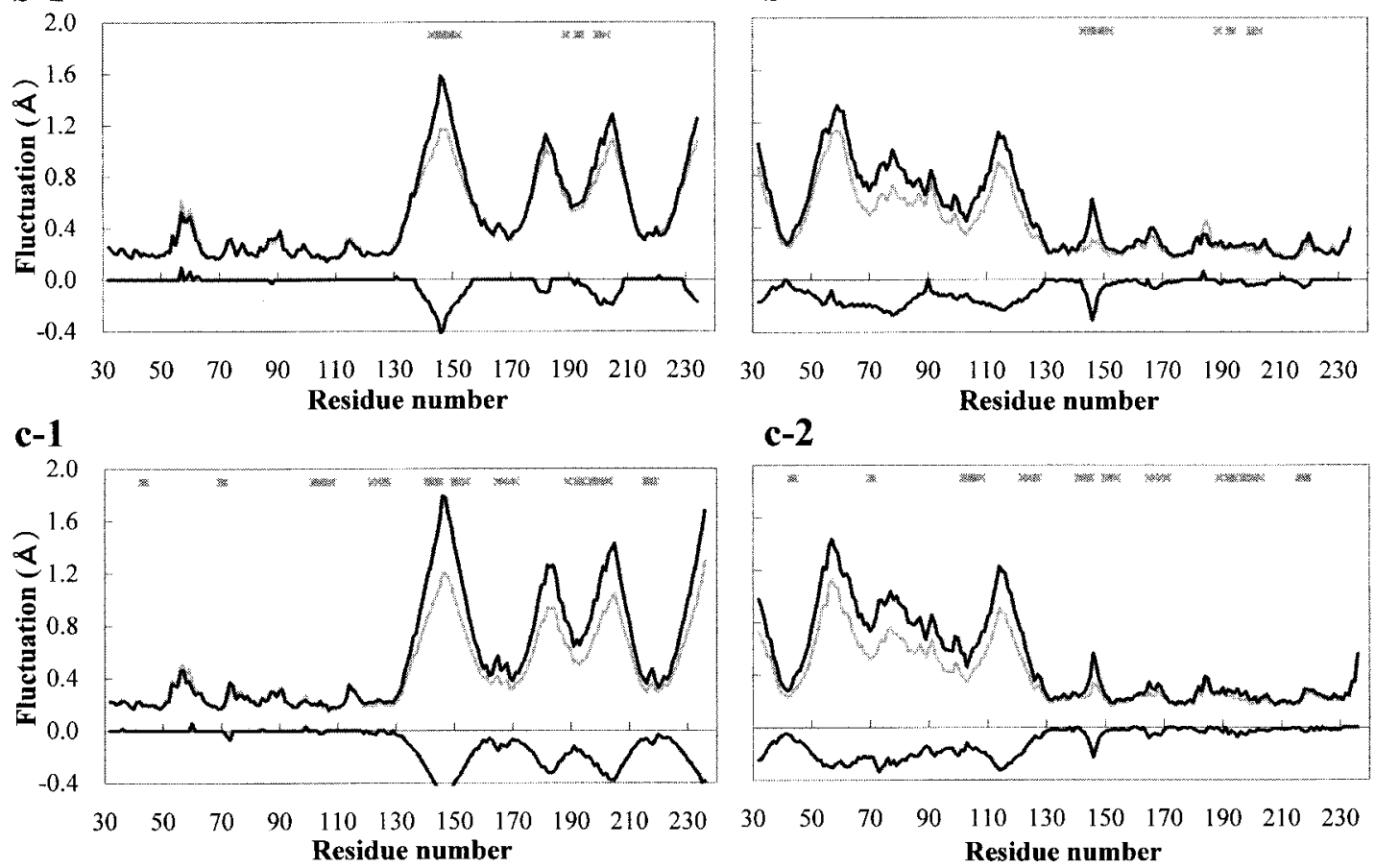

Fig. 4. Fluctuation of $\mathrm{C} \alpha$ Atoms of hGHbps in Free hGHbps, in the hGH-bp1 Heterodimer Complex and in the hGH-bp1-bp2 Ternary Complex When the Center of Gravity Is on Each Domain

Fluctuations were calculated under the condition that the center of gravity was on the N-terminal domain (a-1, b-1, c-1) and the C-terminal domain (a-2, b-2, c-2), respectively. Specifications of (a-1) and (a-2), (b-1) and (b-2), (c-1) and (c-2) are same as those of Figs. 3(a), (b) and (c), respectively. In all figures, the fluctuations, the significant differences and the contact residues are calculated in the same way as those of Fig. 2. 
bp1-bp2, respectively. Consequently, we should qualitatively discuss the difference between the fluctuations of free molecules and those of complex molecules.

The fluctuations of hGH decreased only in the contact regions, but those of hGHbps decreased in almost all residues. This difference in the fluctuations between hGH and hGHbps was further investigated. As hGHbps have the N- and C-terminal domains, fluctuations of $\mathrm{C} \alpha$ atoms were calculated under the condition that the center of gravity was on each domain in the second calculations, though in the first calculations (Fig. 3), the center of gravity was on the entire hGHbp. These fluctuations of $\mathrm{C} \alpha$ atoms of bp 1 in free form, the hGH-bp1 heterodimer complex and the hGH-bp1-bp2 ternary complex, and of bp2 in free form and the hGHbp1-bp2 ternary complex are shown in Fig. 4. In same way as the first calculations in Fig. 3, the fluctuations of hGHbps before and after forming the complexes were also compared in the second calculations in Fig. 4. After binding, most of the domain fluctuations of hGHbps having their center of gravity on their self domain decreased only in the contact regions with the bound proteins (Fig. 4).

The fluctuations in the first calculations included the motions within two domains, while those of the second calculations only included the motions within each domain. After binding to free hGH of free bp1, the fluctuations of bp1 in the first calculations (Fig. 3a) decreased at many residues in addition to the contact regions, and the fluctuations in the second calculations (Figs. 4a-1, a-2) decreased in relation to the contact regions of the domain on which the center of
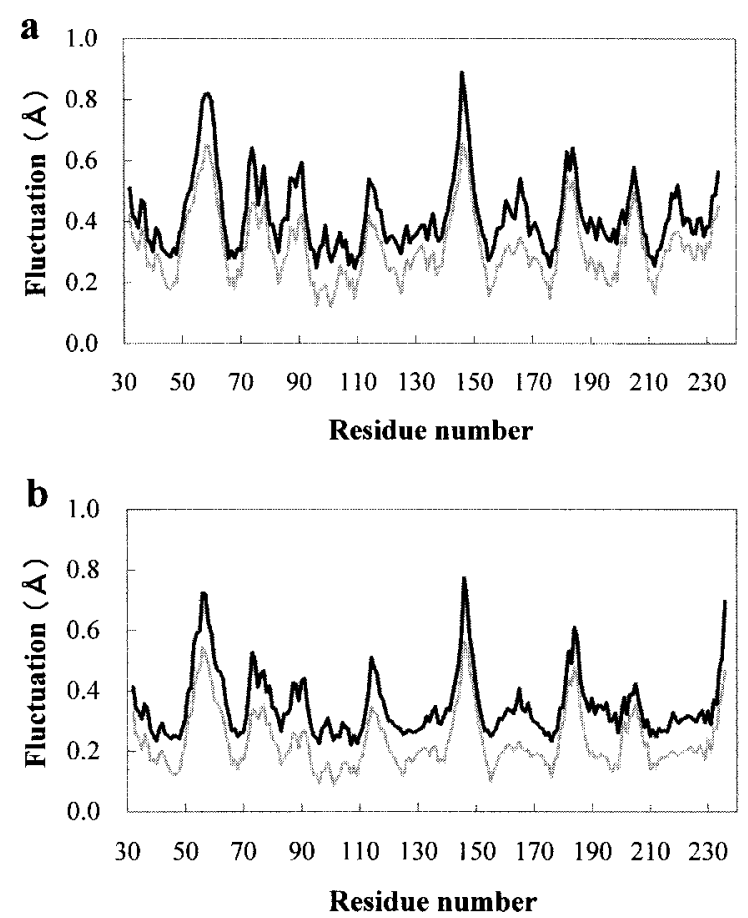

Fig. 5. Fluctuations of $\mathrm{C} \alpha$ Atoms of hGHbps in All Frequency Modes and in the Three Lowest Frequencies

Thick line shows fluctuations of the sum of the contributions from all frequency modes. Thin line shows fluctuations of the sum of the contributions from the modes with the three lowest frequencies. (a) bp1 in free form. (b) bp2 in free form.
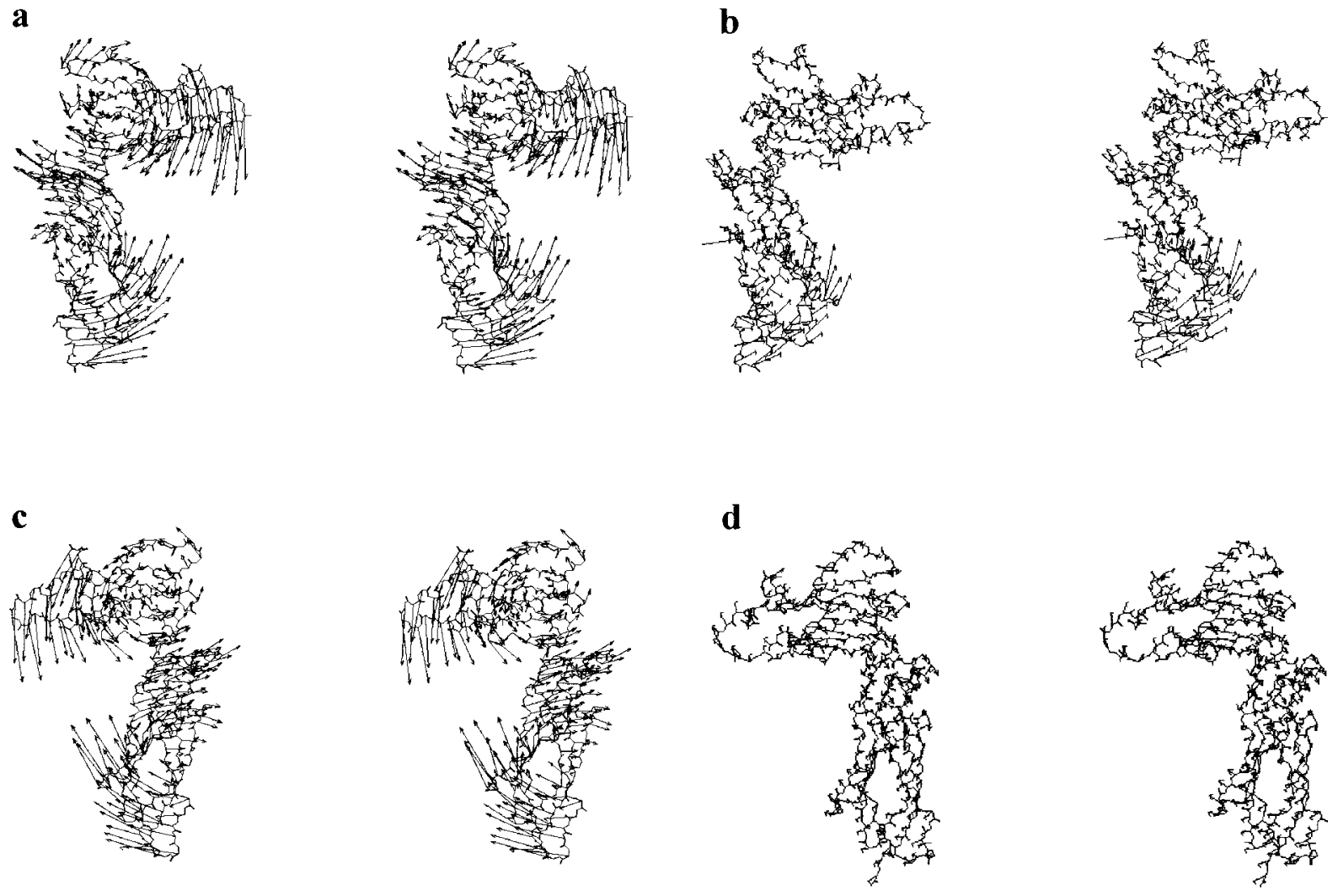

Fig. 6. Stereo Drawing of the Atomic Displacement Vectors of hGHbps

The vector reflects the scale and shows only one side of the motion; that is, each residue actually also has an opposite direct and the same scale motion. All vectors are in the lowest mode in the structure energetically optimized. (a) bp1 in free form. The frequency is $2.50 \mathrm{~cm}^{-1}$. (b) bp1 in the hGH-bp1 heterodimer complex. The frequency is $2.50 \mathrm{~cm}{ }^{-1}$. (c) bp2 in free form. The frequency is $3.47 \mathrm{~cm}^{-1}$. (d) bp2 in the hGH-bp1-bp2 ternary complex. The frequently is $2.41 \mathrm{~cm}^{-1}$. 
gravity was placed. After binding to free bp2 of the hGH-bp1 heterodimer complex, the fluctuation-decreasing regions of bp1 in the first calculations (Fig. 3b) roughly matched those in the second calculations (Figs. 4b-1, b-2). In brief, the inter-domain motion of bp1 was not observed. These results showed that only after binding to hGH were the inter-domain motions of bp 1 almost suppressed. After binding to the hGH-bp1 heterodimer complex of free bp2, the fluctuations of bp2 in the first calculations (Fig. 3c) decreased at many residues in addition to the contact regions, and the fluctuations in the second calculations (Figs. 4c-1, c2) decreased in relation to the contact regions of the domain on which the center of gravity was placed. These results showed that the inter-domain motions of bp 2 decreased after binding to the hGH-bp1 heterodimer complex.

Inter-domain Motions of hGHbps As shown in Figs. 3 and 4, the inter-domain motions of hGHbps greatly decreased after binding to hGH.

It has been thought that large-scale motions in proteins are described by a small number of the lowest frequency principal components. ${ }^{27,28)}$ Therefore, the low frequency modes were focused on hGH and hGHbps. We found that fluctuations of the sum of these three lowest frequencies of hGHbps had similar features to the fluctuations of the sum of all the frequency modes (Fig. 5). These results therefore indicated that the three lowest frequencies are the chief contributors to the total fluctuation. In free hGHbps, the characteristic domain motions between the $\mathrm{N}$ - and $\mathrm{C}$-terminal domains were observed on the three lowest frequency modes in all 10 optimized structures. However, in the complex forms, such interdomain motions were greatly suppressed (Fig. 6). The stereo views in Fig. 6 are placed in the same direction as the X-ray structure as shown in Fig. 1. The normal mode of free bp 1 is very similar to that of free bp2, though the fluctuations of free bp 1 and bp2 are different. Nevertheless, the modes such as inter-domain motions may be insensitive to changes in structure. It was shown that the inter-domain motions were dominant factor in the total fluctuation, and also that the inter-domain motions were decreased by the formation of the complexes.

In a previous paper, ${ }^{18)}$ we reported that the inter-domain motions of human blood coagulation factor (VIIa) were also decreased in complex formation with human tissue factor (sTF). It is interesting for our understanding of protein dynamics that a similar inter-domain phenomenon was found in hGHbps forming complexes with hGH.

\section{Conclusion}

NMA of hGH and hGHbps demonstrated a noteworthy finding for protein interactions in terms of dynamics. hGHbps clearly instituted the inter-domain motion between the $\mathrm{N}$ - and $\mathrm{C}$-terminal domains in free forms. In the complex formations wherein hGH binds to bp1 and sequentially, the hGH-bp1 heterodimer complex binds to $\mathrm{bp} 2$, we found that the inter-domain motions of hGHbps markedly decreased after binding to hGH. It is suggested that hGH regulates the inter-motions of hGHbps.

Acknowledgments We thank Masaaki Adachi of Kitasato University for helpful discussions. This work was supported by a Grant-in-Aid for Scientific Research on Priority Areas (C) "Genome Information Science" from the Ministry of Education, Culture, Sports, Science and Technology of Japan.

\section{References}

1) Paladini A. C., Pena C., Poskus E., CRC Crit. Rev. Biochem., 15, 2556 (1983).

2) Cunningham B. C., Ultsch M., De Vos A. M., Mulkerrin M. G., Clauser K. R., Wells J. A., Science, 254, 821—825 (1991).

3) Argetsinger L. S., Campbell G. S., Yang X., Witthuhn B. A., Silvennoinen O., Ihle J. N., Carter-Su C., Cell, 74, 237-244 (1993).

4) Carter-Su C., Schwartz J., Smit L. S., Annu. Rev. Physiol., 58, 187207 (1996).

5) Fuh G., Cunningham B. C., Fukunaga R., Nagata S., Goeddel D. V., Wells J. A., Science, 256, 1677-1680 (1992).

6) Higo J., Umeyama H., J. Theor. Biol., 186, 477-490 (1997).

7) de Vos A. M., Ultsch M., Kossiakoff A. A., Science, 255, 306-312 (1992).

8) Murzin A. G., Conte L. L., Ailey B. G., Brenner S. E., Hubbard T., Chothia C.: 〈http://scop.mrc-lmb.cam.ac.uk/scop//, Structural Classification of Proteins, 15 May, 2002.

9) Murzin A. G., Brenner S. E., Hubbard T., Chothia C., J. Mol. Biol., 247, 536-540 (1995).

10) Bazan J. F., Proc. Natl. Acad. Sci. U.S.A., 87, 6934-6938 (1990).

11) Cosman D., Lyman S. D., Idzerda R. L., Beckmann M. P., Park L. S., Goodwin R. G., March C. J., Trends Biochem. Sci., 15, 265-270 (1990).

12) Ishida H., Jochi Y., Kidera A., Proteins, 32, 324-333 (1998).

13) Takeda-Shitaka M., Kamiya K., Miyata T., Ohkura N., Madoiwa S., Sakata Y., Umeyama H., Chem. Pharm. Bull., 47, 322-328 (1999).

14) Shen Y., Kong Y., Ma J., Proc. Natl. Acad. Sci. U.S.A., 99, 19491953 (2002)

15) Nojima H., Takeda-Shitaka M., Kurihara Y., Adachi M., Yoneda S., Kamiya K., Umeyama H., Chem. Pharm. Bull., 50, 1209-1214 (2002).

16) Hayward S., Kitao A., Berendsen H. J., Proteins, 27, 425-437 (1997).

17) Hayward S., Berendsen H. J., Proteins, 30, 144-154 (1998).

18) Soejima K., Kurihara Y., Kamiya K., Umeyama H., FEBS Lett., 463, 19-23 (1999).

19) Berman H. M., Westbrook J., Feng Z., Gilliland G., Bhat T. N., Weissig H., Shindyalov I. N., Bourne P. E., Nucleic Acids Res., 28, 235242 (2000).

20) Kajihara A., Komooka H., Kamiya K., Yoneda T., Yoneda S., Nakamura M., Shimizu T., Umeyama H., J. Lipid. Mediat. Cell Signal, 9 , 185-196 (1994).

21) Kaneko H., Kuriki T., Honda S., Takada T., Takata H., Yanase M., Okada S., Umeyama H., Shimada J., Res. Comm. Biochm. Cell Mol. Biol., 2, 69-90 (1998).

22) Sumikawa H., Suzuki E., Fukuhara K., Nakajima Y., Kamiya K., Umeyama H., Chem. Pharm. Bull., 46, 1069-1077 (1998).

23) Weiner S. J., Kollman P. A., Case D. A., Singh U. C., Ghio C., Alagona G., Ptofeta S., Jr., Weiner P., J. Am. Chem. Soc., 106, 765-784 (1984).

24) Sundstrom M., Lundqvist T., Rodin J., Giebel L. B., Milligan D., Norstedt G., J. Biol. Chem., 271, 32197-32203 (1996).

25) Clackson T., Ultsch M. H., Wells J. A., de Vos A. M., J. Mol. Biol., 277, 1111-1128 (1998).

26) Eckart C., Physical Review, 47, 552-558 (1935).

27) Go N., Noguti T., Nishikawa T., Proc. Natl. Acad. Sci. U.S.A., 80, 3696-3700 (1983).

28) Hayward S., Kitao A., Go N., Protein Sci., 3, 936-943 (1994).

29) Akahane K., Nagano Y., Umeyama H., Chem. Pharm. Bull., 37, 8692 (1989). 